\title{
Ultimate Physical Reality of the Universe
}

\section{Moolathurai Rangasamy Vijayakumar}

Bharathiar University, Coimbatore, India

Email: mrvk1954@googlemail.com

How to cite this paper: Vijayakumar, M.R. (2018) Ultimate Physical Reality of the Universe. Journal of Modern Physics, 9, 387-394.

https://doi.org/10.4236/jmp.2018.93027

Received: December 4, 2017

Accepted: February 4, 2018

Published: February 7, 2018

Copyright (c) 2018 by author and Scientific Research Publishing Inc. This work is licensed under the Creative Commons Attribution International License (CC BY 4.0).

http://creativecommons.org/licenses/by/4.0/

\begin{abstract}
According to modern concepts, space continues in all directions, and has no known limits. The sun and billions of other stars make up a giant galaxy whirling through space. Countless other galaxies are scattered throughout space as far as man can see with the largest telescopes. The Newtonian universe, Minkowski universe, Einstein's General Relativistic Universe and the Big-Bang and Steady State models deal with creation and evolution of the universe. Following Einstein, Milne, Friedman, Lemaitre, De Sitter, Bondi and Gold, Hoyle, Narlikar and Brons Dicke have given their own models and tried to explain the nature of the universe. All the above mentioned concepts and models are not able to explain the Ultimate Physical Reality of the Universe. Here I suggest for the first time, the Ultimate Physical Reality of the Universe by explaining that there is no separate "space" without the presence of matter. I give the answer for the unexplained puzzling question: "What the universe expands into?" and explain that the universe is just closed and finite. I explain that the universe is ever expanding. I also give answers to important unanswered questions so far.
\end{abstract}

\section{Keywords}

Ultimate, Reality of the Universe

\section{Introduction}

Following Einstein, Milne, Friedman, Lemaitre, De Sitter, Bondi and Gold, Hoyle, Narlikar and Brons-Dicke have tried to explain the nature of the universe. All the above mentioned theories are not able to explain the Ultimate Physical Reality of the Universe.

Here we show for the first time, the Ultimate Physical Reality of the Universe by explaining that there is no separate "space" without the presence of matter. We give the answer for the unexplained puzzling question: "What the universe expands into?" and explain that the universe is just closed and finite. We also 
explain that the universe is ever expanding.

\section{What Is Space?}

According to classical physics Roger Penrose in his book "The Emperor's New Mind" has given the idea that there is space-time which is playing a primary role as the arena for all the varied activity of physics [1].

Space by itself and time by itself has no meaning at all but the union of these two i.e., space-time only brings out the meaning in reality [2]. "Space-time" diagrams are often referred by scientists only for calculating and explaining the events that are continuously taking place in the universe.

Einstein's general relativity implies space-time and matter are indistinguishable. To "measure" or realize the properties of space, we need matter, i.e., at least exchange light signals between points. But these signals have energy. Space-time is just a tool for recording or book keeping of events, so called arithmetization of events, using 3 co-ordinates of space and one of time. For example say, moon is 4 Lakh kilometers across. Alfa Centauri the nearest star to our earth is 4.3 light years across. If you tell the distance, there arises the space concept. So "space" is a derived concept and it is not a basic entity. Points are derived by intersection of null events (light-ray for example). Events have four momenta associated with them (three momentum co-ordinates and one energy coordinate). So events create and distort space-time, without events (involving particles) one cannot construct a space-time. So there is no separate "space" without the presence of matter.

\section{Universe Is Just Closed and Finite}

Newton's concept of absolute space was criticized by many authors including Bishop Berkeley and especially by Ernst Mach. Mach pointed out that inertia (mass) of any particle in the universe arises from the gravitational interaction of the distant matter spread out with the local particle. This puts a constraint on the nature of space-time itself, as emphasized by Diche, Hoyle-Narlikar etc. A simple argument has to do with inertial forces in rotating frames. Considering a rotating platform, an observer in this frame feels centrifugal and other inertial forces. What is the origin of these forces? One can imagine the platform to be at rest and the rest of the universe going around it (relative motion). In that case, all the distant matter would exert gravitational force on the platform and this can be shown equivalent to the inertial force felt by the observer. So the origin of inertia in this Machian picture is due to the forces exerted by the distant matter spread out [3].

We can give a simple equation to describe this: Suppose $m$ is the mass of the local particle. Its energy is $m c^{2}$. In this picture the origin of this is due to the gravitational interaction of all other particles in the universe (say a total mass of $\mathrm{M}$, which is the sum total of all masses $m, M=\sum m$ ). If $R$ is the radius over which this total mass is distributed, then the gravitational energy acting on the local 
mass $m$ is:

$$
E_{G}=\frac{G M m}{R} \text {, This should equal } \mathrm{mc}^{2} . \therefore m c^{2}=\frac{G M m}{R} \text {, or we get a constraint }
$$

on the mass and radius $R$ of the universe, as: $\frac{G M}{R}=c^{2}$ where $c$ is the velocity of light.

This suggests that the universe should be closed and finite. If all masses (inertia) of particles arise from their mutual gravitational interactions then the total energy of the universe is zero. Gravitational binding energy is negative, while inertial mass (energy) is positive. So a closed universe has zero total energy. This is the universe with just the critical density. All recent observations from microwave background anisotropies and from distant exploding stars support this so called $\Omega=1$ (density equal critical density universe).

Useful analogy: Consider a stone is thrown up from the earth's surface.

Kinetic energy of stone $=\frac{1}{2} m V^{2}$.

Gravitational potential energy $=m g h$.

$$
V^{2}=2 g h . \text { Total energy }=\frac{1}{2} m V^{2}-m g h .
$$

Stone keeps going up, till total energy becomes zero, then falls back, pulled by earth's gravity.

$$
\begin{aligned}
& \frac{1}{2} m V^{2}-m g h=0 \\
& \left(V^{2}=2 g h\right)
\end{aligned}
$$

Similarly the universe keeps expanding and if it is at critical density, it is just closed and it is ever expanding (See Figure 1). In open universe, it will not collapse and it is ever expanding. In closed universe, the expanding universe will recollapse after some time. In reality, the universe is just closed and it is ever expanding at nearly the critical rate and it is finite.

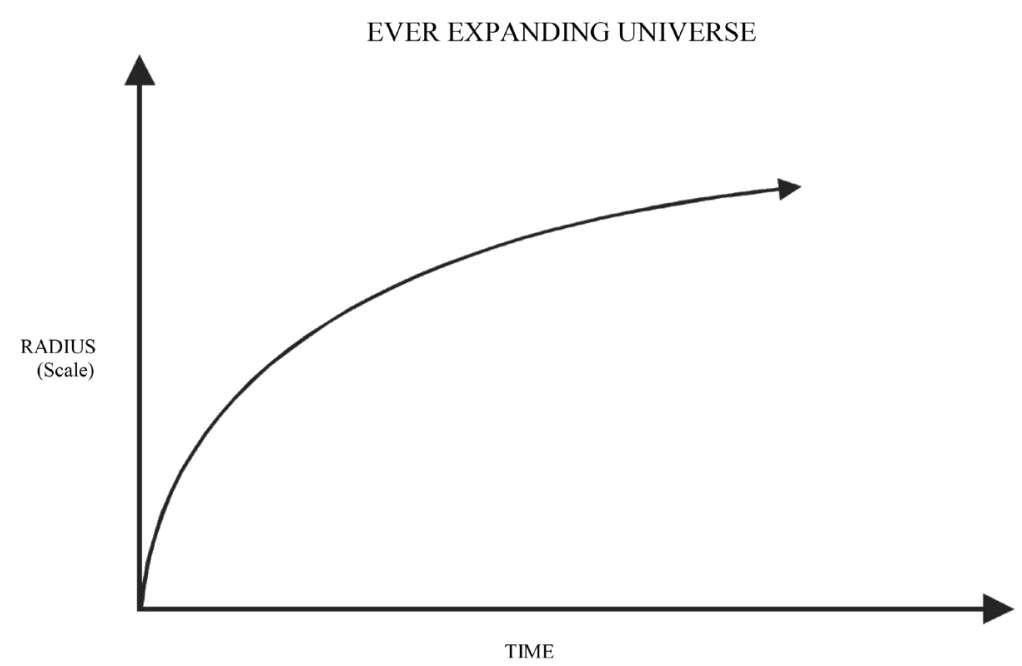

Figure 1. Ever Expanding Universe. Here the universe is just closed and it is ever expanding. 


\section{Expanding Universe}

The geometry of the expanding universe is such that it is static (not expanding) only if there is no matter or energy, so one needs matter or energy for expansion. Thus a static universe is not possible unless it is devoid of all matter. Any presence of matter would cause expansion.

The equation describing this is:

$\frac{\mathrm{d} R / \mathrm{d} t}{R}=\left(\frac{8 \pi G \rho}{3}\right)^{1 / 2}, \rho$ is the average density of the matter distribution in the universe.

Only for $\rho=0$, i.e. no matter, in the left hand side zero, $\mathrm{d} R / \mathrm{d} t$ gives rate of expansion, which vanishes only if $\rho=0$, i.e. zero matter.

Even Einstein made a mistake in his early approach of trying to construct a static universe with only matter. He found he had to introduce an overall repulsion force, the cosmological constant term to balance the energy of matter, so that total energy vanishes. After Hubble discovered universe is expanding, Einstein called this his biggest blunder (of introducing extra term).

\section{What the Universe Expands Into? [4]}

In 1929, the American astronomer Edvin P. Hubble made the incredible discovery that the Universe is expanding. Studying the spectra of galaxies, he showed that the spectra were in general shifted towards the red, indicating that these galaxies were moving away from us [5]. The galaxies all recede away from one another, i.e. the distance between the galaxies is continuously increasing and that the universe is in a state of rapid expansion. The universe that is, fabric of scattered fragments of matter is not inside anything. It is expanding but not into anything. Only due to our ignorance of not knowing the reality, we are asking the questions: "What the universe is in?" and "What the universe expands into?". The universe is just closed and finite, like you can keep going round and round an expanding sphere.

\section{Ever Expanding Universe versus Oscillating Universe}

Since there is acceleration in expansion, the universe should expand forever and ever expanding universe is favoured than oscillating universe. The density required to close the universe, i.e., so called critical density is some twenty five times larger than the baryon density i.e. if the matter were made up of only baryons, the universe would be an open universe, which expand forever, the combined gravity of all the matter not being strong enough to slow down the expansion.

An oscillating universe where universe expands and contracts alternately is a possibility. In this model oscillations occur but with very long periods. However from the thermodynamic point of view, such oscillating models run into problems. This has to do with production of entropy, i.e. increase in the amount of unavailable energy. Formation of black holes (from collapse of massive stars) 
considerably increases the entropy. So the oscillations will not be identical and the probability of getting the same properties of the universe in each cycle is incredibly small. So currently such models are not favoured.

\section{Questions and Answers}

The picture of a universe that started off very hot and cooled as it expanded is in agreement with all the observational evidence that we have today. Nevertheless, it leaves a number of important questions unanswered [6]:

1) Why was the early universe so hot?

Answer: Once we accept the observational fact that the universe is expanding at a definite rate given by the Hubble constant, it follows that the scale factor would have been much smaller at early times, i.e. in the analogy of an expanding balloon, the surface of the balloon would have been much smaller so that the densities of the radiation and matter would have been much higher. If the density is $\rho_{1}\left(R_{1}\right)$ at a radius $R_{1}$ and $\rho_{2}\left(R_{2}\right)$ at a radius $R_{2}$ then $\rho_{1} R_{1}{ }^{3}=\rho_{2} R_{2}{ }^{3}$, as mass is conserved. For radiation, it follows that the temperature and radius scale as $R T=$ constant, $T$ is the temperature.

Radiation would be black body radiation (radiation in complete equilibrium with matter) as it is expanding with the universe. So if the radius of the universe is reduced to one-third, the temperature of the radiation increases 3 times, hundred times smaller, radiation would be hundred times hotter. So as the universe expands, it cools. The relation between temperature $\mathrm{T}$ and time since expansion began, $t$ is given by a well known formula (First derived by George Gamow, more than 60 year ago):

$$
T(\text { in degrees })=\frac{10^{10}}{\sqrt{t \text { sec }}}
$$

So, one second after the universe started expanding Temperature is ten thousand million degrees. One picosecond after expansion began $\left(t=10^{-12} \mathrm{sec}\right.$.) $T=$ $10^{16}$ degrees. This is the TeV energy range which the LHC accelerator at CERN is now probing. It will mimic the conditions in the early universe, to see what new particles would be produced and various new possible phenomena to understand what happened in the beginning period of the universe. So the above formula explains why the early universe was so hot.

It is just an application of thermodynamic of black body radiation to the expanding space-time and predicts that now (14 billion years after expansion) we should be surrounded by radiation of a few degrees Kelvin, which is seen as background microwave cosmic radiation.

2) Why is the universe so uniform on a large scale? Why does it look the same at all points of space and in all directions? In particular, why is the temperature of the microwave background radiation so nearly the same when we look in different directions?

Answer: This is the so called horizon problem. A solution has been provided by one so called INFLATION MODEL. This postulates that the very early un- 
iverse was dominated by the quantum vacuum energy which has negative pressure. Heisenberg's uncertainty principle says that if you squeeze particles closer and closer together their velocities (momenta) would increase, the product position $x$ momentum $=h=$ Planck's constant. The higher momenta would mean that they exert more pressure (like gas molecules hitting a wall), but as the total energy is conserved this pressure would be negative.

Thus $P_{\text {vac. }}=-\rho C^{2}=-n m c^{2}$.

$\rho$ is the density, $\mathrm{C}$ the speed of light. This -ve pressure would make the universe expand exponentially fast, i.e. $R=R_{0} \exp (t c t)$, where $t=$ time, $t c=$ constant and $R_{0}=$ initial radius.

This briefly implies that a single region in causal contact (i.e. within light horizon, $c t$ ) would blow up rapidly, giving it the same uniformity all over.

This rapid expansion would imply "memory loss" of initial conditions, i.e. something like terminal velocity in a fluid. Suppose a body is falling in a viscous medium at high velocity. After a long time it will reach a uniform speed called terminal speed irrespective of its initial speed or position. So a collection of particles at all different velocities, would end up after a sufficiently long time with the same uniform speed. Similarly here different parts or patches of the expanding region would tend to have the same uniform properties.

3) Why did the universe start out with so nearly the critical rate of expansion that separates models that recollapse from those that go on expanding forever, so that even now, ten thousand million years later, it is still expanding at nearly the critical rate? If the rate of expansion one second after the Big Bang had been smaller by even one part in a hundred thousand million, the universe would have recollapsed before it ever reached its present size.

Answer: This is the so called FLATNESS PROBLEM, i.e. the universe being now close to the critical density, would have been arbitrarily close to the critical density at early epochs. Thus one second after the expansion began, it would have been close to the critical density to within one part in $10^{20}$. This is also explained by the so called INFLATION MODEL as given above in Answer 2.

The exponential expansion due to the dominance of the quantum vacuum, would result in a universe expanding at exactly the critical density. This implies that the kinetic energy and potential energy are exactly equal at all epochs and that the total energy of the universe is ZERO and has been ZERO right from the beginning.

In Inflationary Picture, the universe was created from quantum vacuum, with total energy zero. Pressure just cancels the energy density $P+\rho c^{2}=O . P=-\rho c^{2}$, drives exponential expansion. As volume $\mathrm{V}$ increases, pressure energy (-PV) becomes more and more negative and to balance this particles must be created with positive energy, i.e. $+\rho \mathrm{c}^{2} \mathrm{~V}=-\mathrm{PV}$ so that total $\mathrm{PV}+\rho \mathrm{c}^{2} \mathrm{~V}=\mathrm{O}$. This is also called creation ex-nihilo, i.e. creation from nothing and as A GUTH, one of the proponent of inflation model noted "The universe is a FREE LUNCH". So this seems to explain three things: 
a) Why the universe started expanding;

b) How was all the matter created in the beginning;

c) Why it is so uniform on a large scale.

This prediction of expansion at exact critical density seems to be consistent with many observations recently.

4) Despite the fact that the universe is so uniform and homogenous on a large scale, it contains local irregularities, such as stars and galaxies. These are thought to have developed from small differences in the density of the early universe from one region to another. What was the origin of these density fluctuations?

Answer: The structures in the universe, are supposed to have grown from density fluctuations. Again the inflation model predicts that quantum fluctuations in a scalar field would grow in an expanding universe and these fluctuations even if just one part in $10^{5}$ would be stretched with expansion and cause anisotropies in the microwave background. These anisotropies have now been seen in COBE and WMAP satellites. However to form the largest structures we need vast amounts of Dark Matter. We still have the singularity problem. It is supposed that classical general relativity may not be valid around $t \rightarrow 0$, so quantum effects could avoid such infinite densities, etc. So there would be lower limits to time (quantified space-time) and upper limits to physical quantities like temperature etc. This is still a problem which specialists are trying to resolve.

\section{Discussion and Findings}

The entire matter (and energy) with its fields (i.e. universe) is not inside anything. Universe means-just fabric of scattered fragments of matter with energy and their fields. The fields are given very specifically-the electromagnetic field being subject to the Maxwell equations and the gravitational field to the Einstein equation [7]. The universe is finite. It is ever expanding. It expands but not into anything. Since there is acceleration in expansion, the universe should expand forever and ever expanding universe is favoured than oscillating universe.

\section{Conclusions}

There is no separate "space" without the presence of matter. Space-time is just a tool for recording or book keeping of events. In recent approaches, space-time is a derived concept and it is not a basic entity. So events create and distort space-time and without events (involving particles), one cannot construct a space-time. The universe consists of matter and energy only.

So far, various models and concepts of the universe have been proposed. But they have not given the ultimate physical reality of the universe and the answer for "What the universe expands into?"-An unexplained puzzling question. This concept makes the question meaningless by showing that universe expands, but not into anything. This concept shows the ultimate physical reality of the universe and the universe is finite and it is ever expanding. We have given answers for important unanswered questions so far. 


\section{References}

[1] Penrose, R. (1989) The Emperor's New Mind. Oxford University Press, Oxford, 280.

[2] Harrison, E. (2003) Masks of the Universe. 2nd Edition, Cambridge University Press, New York, 150. https://doi.org/10.1017/CBO9780511536564

[3] Bondi, H. (1961) Cosmology. Cambridge University Press, Cambridge.

[4] Peacock, J.A. (1999) Cosmological Physics. Cambridge University Press, Cambridge, 86 .

[5] Gleiser, M. (1998) Contemporary Physics, 39, 239-253. https://doi.org/10.1080/001075198181937

[6] Hawking, S. (1988) A Brief History of Time. Bantam Press, New York, 127. https://doi.org/10.1063/1.2811637

[7] Penrose, R. (1989) The Emperor's New Mind. Oxford University Press, Oxford, 280. 